\title{
Chromophores from hexeneuronic acids: chemical behavior under peroxide bleaching conditions
}

\author{
Nele Sophie Zwirchmayr • Takashi Hosoya - Hubert Hettegger • \\ Markus Bacher - Karin Krainz • Thomas Dietz • Ute Henniges • \\ Antje Potthast $\cdot$ Thomas Rosenau
}

Received: 9 April 2017 / Accepted: 1 July 2017/Published online: 13 July 2017

(C) The Author(s) 2017. This article is an open access publication

\begin{abstract}
Hexeneuronic acids (HexA) are a major cause of discoloration (yellowing/brightness reversion) in pulps from xylan-containing wood, being generated from the xylan's 4- $O$-methylglucuronic acid residues. The HexA-derived chromophores, whose identification and structure confirmation have been described in the previous part of this series (Rosenau et al. in Cellulose, 2017), were subject to conditions of peroxide bleaching, i.e. treatment with hydrogen
\end{abstract}

This work is dedicated to Dr. Hans-Ulrich Suess, formerly Degussa and Degussa-Evonik, who devoted his whole life to the understanding of chromophore formation in cellulosic pulps and bleaching phenomena.

Chromophores in cellulosic materials. Part XVII.

N. S. Zwirchmayr · T. Hosoya · H. Hettegger .

M. Bacher · U. Henniges · A. Potthast · T. Rosenau ( $₫)$

Department of Chemistry, Division of Chemistry of

Renewable Resources, BOKU University Vienna,

Muthgasse 18, 1190 Vienna, Austria

e-mail: thomas.rosenau@boku.ac.at

K. Krainz

Air Liquid Vienna, Vienna, Austria

T. Dietz

Evonik-Degussa, Rodenbacher Chaussee 4,

63457 Hanau-Wolfgang, Germany

T. Rosenau

Johan Gadolin Process Chemistry Centre, Åbo Akademi

University, Porthansgatan 3, 20500 Turku, Finland peroxide in alkaline medium. These chromophores, ladder-type oligomers of mixed aromatic-quinoid and mixed furanoid-benzoid character, are degraded relatively quickly to one major product, 2,5-dihydroxy[1,4]-benzoquinone (DHBQ), and a minor component, 2,5-dihydroxyacetophenone (DHA). These two compounds, which have already been identified as two of the three key chromophores (besides 5,8-dihydroxy[1,4]-naphthoquinone, DHNQ) in aged cellulosics, are potent chromophores themselves and are subsequently more slowly degraded to non-colored degradation products, according to pathways already described in previous parts of this series. The occurrence of DHBQ and DHA in the bleaching treatment of the HexAderived chromophores establishes the link between HexA chemistry and the key chromophore classes of residual chromophores found in aged cellulosic materials.

Keywords 2,5-Dihydroxy-[1,4]-benzoquinone · 2Furancarboxylic acid · A-stage · Furan - Cellulose . Chromophores · Hexeneuronic acids · Ladder-type oligomers $\cdot$ Pulp bleaching $\cdot$ Pulp $\cdot$ Yellowing

\section{Introduction}

Hexeneuronic acids (HexA) are known as contributors to yellowing and brightness reversion in cellulosic hardwood pulps. They originate from glucuronoxylan, 
the major component of the hardwood's hemicelluloses, whose 4-O-methyl glucuronic acid moieties eliminate methanol under the quite drastic pulping conditions (Clayton 1963; Suess 2010). The resulting 4-deoxy- $\beta$-L-threo-hex-4-enopyranosiduronic acid (HexA) moieties are rather unstable and readily further converted to degradation products. In acidic washing stages this conversion is deliberate and aims at depleting the pulp of those moieties. In brightness reversion, residual HexA units generate chromophores that negatively influence the optical properties of the celluloses.

The preceding part of this series has reported the chemical structure of the HexA-derived chromophores and their detailed analytical characterization for the first time. The chromophores are mixed aromatic/quinoid and furanoid/benzoid ladder-type oligomers consisting of largely unchanged 2-furanoyl units (see Scheme 1). The five compounds described account for $90 \%$ of the chromophoric material isolated by the CRI procedure (Rosenau et al. 2004, 2007; Korntner et al. 2015). The linkage pattern of these units and the formation mechanisms of the chromophores have been clarified by means of ${ }^{13} \mathrm{C}$-isotopic labeling. Mono- ${ }^{13} \mathrm{C}$-labeled HexA model compounds produce characteristic ${ }^{13} \mathrm{C}$-isotopic splitting patterns in the chromophore products, which allowed identification of the positions and connections of their individual 2-furanoyl building blocks. In particular the occurrence of homonuclear ${ }^{1} J_{\mathrm{C}, \mathrm{C}}$ couplings in the NMR spectra is proof of direct carbon-carbon bonds between two labeled atoms.
Considering that major portions of chemicals, energy and technology costs in the pulp and paper industries are due to bleaching, i.e. removal of chromophores from pulp to reach target brightness, the question how chromophores can be destroyed best is not only of scientific relevance, but also of high economic interest. "Best" chromophore destruction does thus not only mean a protocol that leaves the cellulose unaffected and unharmed, but also an approach which is economically as viable as possible. Before entering such considerations and possible process optimization, it was necessary, of course, to study the general chemistry of the HexA-derived chromophores, and in particular their bleaching response.

The double bond present in HexA allows electrophilic attacks, e.g. by the bleaching agents chlorine dioxide $\left(\mathrm{ClO}_{2}\right)$ or ozone $\left(\mathrm{O}_{3}\right)$, but at the same time nucleophilic attacks, e.g. by hydrogen peroxide. This Janus character is due to the peculiar HexA structure with the double bond being "either" part of an electron-rich vinyl ether or an electron-deficient Michael system. In delignification steps with oxygen only, HexA is not degraded (Suess 2010; Buchert et al. 1995). Consequently, the readily accessible double bond makes HexA a major consumer of bleaching chemicals. Removing it prior to bleaching can reduce the amount of bleaching chemicals needed (Clayton 1963; Chakar et al. 2000). Pretreatments, from which HexA-rich pulps (i.e. hardwood pulps) will especially benefit, are hot acid washes. These allow far-reaching destruction of HexA, as performed with treatment<smiles>O=C1c2ccoc2C(=O)c2occc21</smiles><smiles>[Z]c1cc2oc(C(=O)O)cc2c(=O)c2oc(C(=O)O)cc12</smiles><smiles>O=C1c2ccoc2C(=O)c2c1oc1c(C(=O)O)ccc(O)c21</smiles><smiles>O=C(O)c1ccc(O)c2c1oc1c(C(=O)O)ccc(O)c12</smiles><smiles>O=c1c2ccoc2c(=O)c2c1oc1c(=O)c3oc4c(=O)c5occc5c(=O)c4c3c(=O)c12</smiles>

Scheme 1 Chemical structures of the five isolated HexA-derived chromophores 
times of $2 \mathrm{~h}$, temperatures greater than $90{ }^{\circ} \mathrm{C}$ and $\mathrm{pH}$ values $<3$ (Suess 2010). Under these conditions, HexA is mainly degraded to formic acid, 2-furancarboxylic acid and 5-formyl-2-furancarboxylic acid, all colorless and water-soluble substances, thus they are easy to remove by extraction (Teleman et al. 1996) (see also previous parts of this series). Varying sequences of hot acid hydrolysis and $\mathrm{ClO}_{2}$ (D-stage) improved the bleaching results, but the fast, competitive reaction of $\mathrm{ClO}_{2}$ with lignin and the degradation products of HexA still has to be considered (Lachenal and Chirat 1998). Additionally, a compromise has to be found between retention time, increased brightness and molecular weight of the cellulose, as the hot acid stage decreases the molecular weight of the pulp significantly (Suess 2010). Final increases in brightness were achieved by peroxide treatments-followed by ECF-bleaching and/or extractions. The positive outcome can be attributed to both, the hydrolysis and degradation of HexA, and the removal of lignin residues (Suess 2010; Petit-Breuilh et al. 2004). In newer developments in bleaching chemistry the role of catalysis was investigated, where molybdenum with hydrogen peroxide (Azevedo et al. 2011) and tertiary amines in combination with $\mathrm{ClO}_{2}$ /hypochlorous acid (HOCl) (Chenna et al. 2013) have been reported as efficient catalysts for the removal of HexA.

\section{Materials and methods}

\section{General}

Commercial chemicals were of the highest grade available and were used without further purification. ${ }^{1}$ Reagent-grade solvents were used for all extractions and workup procedures. Distilled water was used for all aqueous extractions and for all aqueous solutions. $n$-Hexane, diethyl ether, ethyl acetate and petroleum ether used in chromatography were distilled before use. All reactions involving non-aqueous conditions were conducted in oven-dried $\left(140{ }^{\circ} \mathrm{C}\right.$, overnight $)$ or flame-dried glassware under an inert (argon or nitrogen) atmosphere. TLC was performed using Merck silica gel $60 \mathrm{~F}_{254}$ pre-coated glass plates. Flash

\footnotetext{
${ }^{1}$ Note: this part on general analysis is equal to the corresponding description part in the preceding part of this series as the work is closely related-no unlawful duplication is intended.
}

chromatography was performed using Baker silica gel (40 $\mu \mathrm{m}$ particle size). All products were purified to homogeneity by TLC/GC-MS analysis. The use of brine refers to saturated $\mathrm{NaCl}_{(\mathrm{aq})}$. All given yields refer to isolated, pure products.

${ }^{1} \mathrm{H}$ NMR spectra were recorded at $400 \mathrm{MHz}$ for ${ }^{1} \mathrm{H}$ and at $100 \mathrm{MHz}$ for ${ }^{13} \mathrm{C} \mathrm{NMR}$ in $\mathrm{CDCl}_{3}$ at room temperature, if not given otherwise. Chemical shifts, relative to tetramethylsilane (TMS) as the internal standard, are given in $\delta \mathrm{ppm}$ values, and coupling constants are given in $\mathrm{Hz} .{ }^{13} \mathrm{C}$ peaks were assigned by means of high-resolution $1 \mathrm{D}-{ }^{13} \mathrm{C}$, APT, HSQC and HMBC spectra.

GC-MS analysis was carried out on an Agilent $6890 \mathrm{~N} / 5975 \mathrm{~B}$ instrument in the ESI $(70 \mathrm{eV})$ ionization mode. UV spectra were measured with a Perkin Elmer Lambda $35 \mathrm{UV} / \mathrm{V}$ is spectrometer. IR spectra were recorded with a Perkin Elmer FT-IR Spectrometer Frontier equipped with a Universal ATR Sampling Accessory. Each sample was scanned in quadruplicate from 4000 to $650 \mathrm{~cm}^{-1}$ with a resolution of $4 \mathrm{~cm}^{-1}$. Melting points, determined on a Kofler-type micro hot stage with Reichert-Biovar microscope, are uncorrected. Elemental analyses were performed at the Microanalytical Laboratory of the University of Vienna. All compounds showed satisfactory microanalytical data within the limits of $0.2 \%$.

Treatment of neat HexA-derived chromophores under conditions of peroxide bleaching

The degradation reaction was started by adding a $30 \%$ aqueous solution of hydrogen peroxide $(1.8 \mathrm{~mL})$ to $50 \mathrm{~mL}$ of $0.01 \mathrm{M} \mathrm{NaOH}(\mathrm{pH} 12)$ containing the chromophore in $0.1 \mathrm{mM}$ concentration in a $100 \mathrm{~mL}$ round bottom flask. The solution was preheated at temperatures between $283.15\left(10{ }^{\circ} \mathrm{C}\right)$ and $343.15 \mathrm{~K}$ $\left(70{ }^{\circ} \mathrm{C}\right)$ before addition of the hydrogen peroxide solution. An aliquot of the reaction were transferred into a $5 \mathrm{ml}$ quartz UV cuvette which was placed into a UV spectrometer with a thermostat unit set to the same temperature as the reaction vessel. For GC analysis, a $1 \mathrm{ml}$ aliquot was mixed with $1.2 \mathrm{ml}$ of $0.01 \mathrm{M}$ aqueous $\mathrm{HCl}$. The mixture was freeze-dried and either taken up in $2 \mathrm{~mL}$ of ethanol/THF ( $/ \mathrm{v}=1: 1)$ for $\mathrm{GC}$ analysis or dissolved for NMR analysis.

Degradation of the isotopically labelled chromophores was carried out according to the same procedure using $1.2 \mathrm{~mL} \mathrm{H}_{2} \mathrm{O}_{2}$ solution, $20 \mathrm{ml}$ of 
$0.01 \mathrm{M} \mathrm{NaOH}$ and $1 \mathrm{mM}$ chromophore solutions, but otherwise identical conditions.

Treatment of HexA-derived chromophores on pulp under conditions of peroxide bleaching.

Application of chromophores

The individual chromophore $(0.2 \mathrm{mmol})$ was dissolved in methanol $(8 \mathrm{ml})$ and uniformly sprayed onto $150 \mathrm{~g}$ of dry pulp, resulting in a chromophore concentration of $0.0013 \mathrm{mmol} / \mathrm{g}$ pulp). The solvent was evaporated at room temperature overnight, without applying reduced pressure. The integrity of the chromophores, being stable quinones or stabilized acetophenones, was not changed during the application-evaporation procedure as confirmed by UV and ATR-IR. After application of the chromophores to the pulp, the samples were slightly colored.

\section{Pulp bleaching}

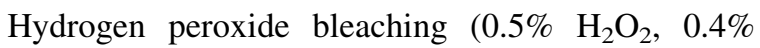
$\mathrm{NaOH}, 70{ }^{\circ} \mathrm{C}, 1 \mathrm{~h}$ ) was run in double-sealed plastic bags in water baths. The impregnated pulps $(50 \mathrm{~g})$ were mixed with water, $\mathrm{NaOH}$ and $\mathrm{H}_{2} \mathrm{O}_{2}$ to $10 \%$ stock consistency $(\mathrm{pH}=12)$, and the suspension was kneaded before incubation of the sealed bags in the water bath. A pulp aliquot (5 g) was taken for handsheet production, which was disintegrated with water to $1 \%$ stock consistency. The $\mathrm{pH}$ of the suspension was adjusted to $\mathrm{pH}=6$ by addition of $\mathrm{H}_{2} \mathrm{SO}_{4}(0.1 \mathrm{M})$. The mixtures were filtrated through a Büchner funnel, washed with deionized water and the remainder dried to obtain handsheets. The combined washings were concentrated to dryness in vacuo at $40{ }^{\circ} \mathrm{C}$, and the remainder used for further analysis (GC-MS, NMR, IR, microanalysis, see above).

Analytical data of the primary products of peroxide bleaching of the HexA-derived chromophores

The following products were obtained when the HexA-derived chromophores (1-5) were subject to standard peroxide bleaching ("P-stage") conditions. The data agree very well with previously published data of the respective compounds (Rosenau et al. 2007, 2011).

2,5-Dihydroxy-[1,4]benzoquinone (DHBQ, 6). ${ }^{1} \mathrm{H}$ NMR (DMSO-d $\left.{ }_{6}\right): \delta 5.81(\mathrm{~s}, 2 \mathrm{H}, \mathrm{CH}), 11.55 \mathrm{ppm}(\mathrm{s}$,
2H, OH). ${ }^{13} \mathrm{C}$ NMR (DMSO-d 6 ): $\delta 105.03$ (CH, d.i.), $170.80 \mathrm{ppm}$ (broad singlett). MS (ESI, -), $\mathrm{m} / \mathrm{z}(\%)$ : 110.9 (42), $138.9\left(100,[\mathrm{M}-\mathrm{H}]^{+}\right)$. Calcd. $\mathrm{C}_{6} \mathrm{H}_{4} \mathrm{O}_{4}$, $\mathrm{M}=140.10$.

2,5-Dihydroxyacetophenone (HAP, 7). ${ }^{1} \mathrm{H}$ NMR $\left(\mathrm{DMSO}_{6}\right): \delta 2.58\left(\mathrm{~s}, 3 \mathrm{H}, \mathrm{CH}_{3}\right), 6.79(\mathrm{~d}, 1 \mathrm{H}$, $\left.{ }^{3} J=8.85 \mathrm{~Hz}\right), \quad 7.00 \quad\left(\mathrm{dd}, \quad 1 \mathrm{H}, \quad{ }^{3} J=8.85 \mathrm{~Hz}\right.$, $\left.{ }^{4} J=3.00 \mathrm{~Hz}\right), 7.19\left(\mathrm{~d}, 1 \mathrm{H},{ }^{4} J=3.00 \mathrm{~Hz}\right), 9.18(\mathrm{~s}$, 1H, 5-OH), $11.88 \mathrm{ppm}(\mathrm{s}, 1 \mathrm{H}, 2-\mathrm{OH}) .{ }^{13} \mathrm{C}$ NMR $\left(\right.$ DMSO-d 6 ): $\delta 27.60\left(\mathrm{CH}_{3}\right), 115.36\left({ }^{\mathrm{Ar}} \mathrm{CH}\right), 118.23$ $\left({ }^{\mathrm{Ar}} \mathrm{CH}\right), 120.11,124.42\left({ }^{\mathrm{Ar}} \mathrm{CH}\right), 149.32,153.76$, $203.92 \mathrm{ppm}(\mathrm{C}=\mathrm{O})$. MS (ESI, -), $\mathrm{m} / \mathrm{z}(\%)$ : 108.0 (25), $150.9\left(100,[\mathrm{M}-\mathrm{H}]^{-}\right)$. Calcd. $\mathrm{C}_{8} \mathrm{H}_{8} \mathrm{O}_{3}, \mathrm{M}=152.15$.

\section{Results and discussion}

Degradation kinetics

It was clear that the identification of the main chromophores derived from HexA units in pulp (see compounds 1-5 in Scheme 1) was quite helpful in bleaching research: while hitherto the bleaching attempts could only rely on reasonable suggestions and assumptions regarding the structure of the chromophores and probable chromophoric motifs of such compounds, the knowledge of the exact chemical structures now allowed targeting these compounds directly. In a first study of their bleaching behavior, we applied standard peroxide bleaching conditions (Pstage) to study the response of those chromophores to such common treatment. While the $\mathrm{pH}$ was kept at 12 , the temperature was mostly lower than under industrial conditions and was also varied-simply to allow us recording kinetics and deriving activation parameters, which would not have been possible at the industrially used temperature of $70-90{ }^{\circ} \mathrm{C}$. As in the two preceding parts of this paper, isotopic labeling $\left({ }^{13} \mathrm{C}\right)$ in combination with nuclear magnetic resonance spectroscopy (NMR) was used as a tool to obtain some insight into the reaction mechanisms. Also ${ }^{13} \mathrm{C}$ isotopomers of the chromophores-compounds that carried ${ }^{13} \mathrm{C}$ labels ( $>99 \%$ isotopic purities) at certain carbon positions-were degraded under bleaching conditions, and the isotopic pattern in the degradation products was used in order to draw conclusions about the underlying mechanistic pathways.

In a first set of experiments, each of the chromophores 1-5 was subject to peroxide bleaching in an 
excess of hydrogen peroxide at $\mathrm{pH} 12$ and $50{ }^{\circ} \mathrm{C}$, the conditions resembling a $\mathrm{P}$-stage in industrial pulp bleaching sequences. The conditions were thus also comparable to the experiments on peroxide bleaching of the cellulosic key chromophores, such as 2,5dihydroxy-[1,4]-benzoquinone (DHBQ), which had recently been studied in detail (Hosoya and Rosenau 2013a, b). As in these previous studies, kinetics of the reaction have been investigated by UV spectroscopy. The $\mathrm{OH}^{-}$concentration and the concentration of hydrogen peroxide were much higher than that of the chromophore, and could be considered constant, so that an involvement of either hydroxyl ions or hydrogen peroxide in the rate-determining step, in addition to the chromophore itself, would result in pseudo-first order overall kinetics.

Just from visual observation, a relatively fast initial color change to blueish was noticed for all five chromophores, followed by a much slower fading of the blue, finally ending up in a colorless solution. When observed by UV spectroscopy (either 340, 400 or $457 \mathrm{~nm}$ ), the initial degradation phase strictly followed first-order kinetics up to a consumption of about $30-40 \%$. This was followed by a "transition region" for which the reaction order could not clearly be assigned - and eventually by another region of firstorder kinetics with lower reaction rate (see Fig. 1). Interestingly, the latter first-order phase had a similar rate constant for all five chromophores $\mathbf{1 - 5}$, while the

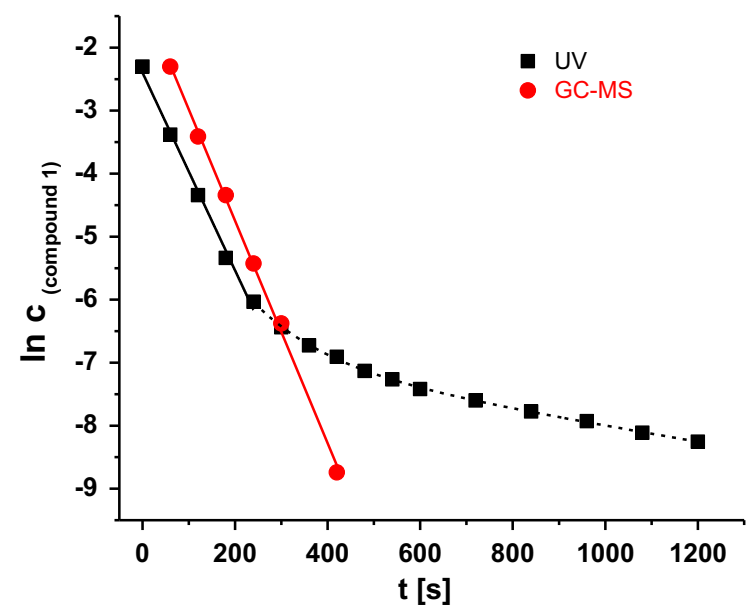

Fig. $1 \ln c$ versus $t$ graphs for the degradation of chromophore $1(0.1 \mathrm{mM})$ by alkaline $\mathrm{H}_{2} \mathrm{O}_{2}$ (excess) at $70{ }^{\circ} \mathrm{C}$. Monitoring by GC shows a steady consumption according to first-order kinetics, while UV measurements shows a first-order initial phase followed by a transition region and a first-order final phase initial first-order phase was clearly different for the five compounds. Evidently, the reaction kinetics changed from a fast to a slower reaction, both being of (pseudo-) first order. Since all conditions remained unchanged during the experiments, an alteration of the reaction mechanism for the same compound (i.e. one compound being first degraded according to one mechanism and later according to another, i.e. different, mechanism) seemed rather unlikely, so we supposed that all five HexA chromophores degraded into the same intermediate which was relatively stable and decomposed more slowly than did the actual starting compounds. The similarity of that intermediate for all compounds would account for the fact that the last degradation phase of the chromophores had always the same rate. To observe the consumption of the HexA chromophores directly by UV was thus rather tricky due to the formation of a colored, UV-absorbing intermediate that interfered with the kinetic measurement and pretended kinetics with three phases of different rates.

When the consumption kinetics were followed by GC-MS instead of UV, it was evident that for all five HexA chromophores the $\ln [\mathrm{c}]$ versus $t$ curve was a straight line over the whole concentration range. The slopes were identical with the respective initial degradation slope measured by UV, within the error of measurement. This is exemplarily shown in Fig. 1 for compound 1, but was true for all five chromophores.

The chromophoric compounds were consumed uniformly according to first-order kinetics, the rate constants evidently being different for each of the five compounds (Fig. 2). The numerical values for the kinetic rate constants retrieved from the slope of the regression lines in Fig. 1 are summarized in Table 1. At $70{ }^{\circ} \mathrm{C}$, the kinetic rate constants for the degradation of compounds 1-5 in alkaline hydrogen peroxide were in the approximate ratio 3:4:25:14:1, i.e. compound 5 was the slowest to be consumed and the degradation of compound 3 was the fastest, being about 25 times more rapid than that of $\mathbf{3}$. Also the half-times for the degradation $\tau_{1 / 2}$ (Table 1) allow for easy comparison of the reaction rates of the individual compounds. While at $70{ }^{\circ} \mathrm{C}$ the starting concentration drops to one half in about $5 \mathrm{~s}$ in the case of compound $\mathbf{3}$, it takes about 2 min for compound $\mathbf{5}$. Note that the half-times for first-order reactions are independent of the starting concentration: $\tau_{1 / 2}=\ln 2 / k$, with $k$ being the rate constants determined from the kinetic measurements. 


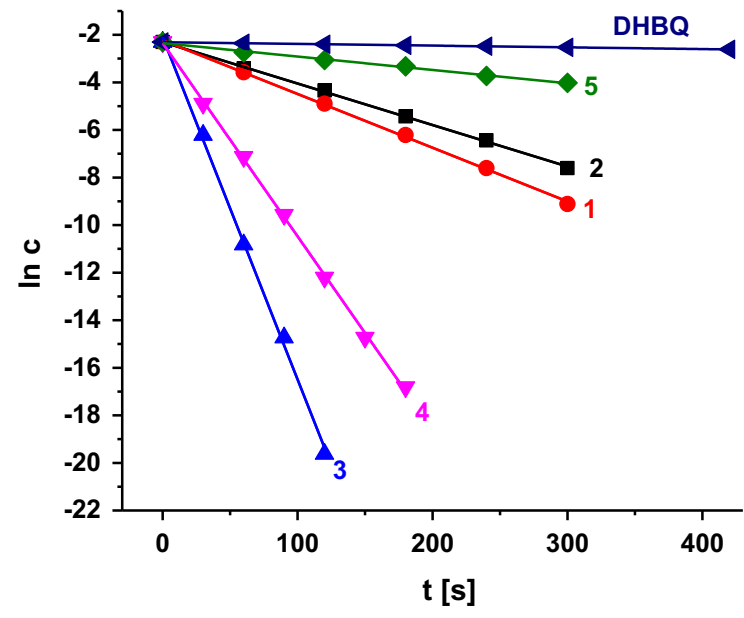

Fig. 2 Degradation of the HexA-derived chromophores 1-5and intermediate DHBQ for comparison-by alkaline $\mathrm{H}_{2} \mathrm{O}_{2}$ at $70{ }^{\circ} \mathrm{C}$ : $\ln c$ versus $\mathrm{t}$ graphs, showing strictly linear correlation for all compounds, indicating first-order kinetics. Measurements for compounds $\mathbf{3}$ and $\mathbf{4}$ stop at 120 and $180 \mathrm{~s}$, respectively, as the chromophores were completely consumed by those times, for the other three compounds kinetics were recorded up to $300 \mathrm{~s}$

In the final phases of the reactions (at consumptions above $70 \%$ as determined by GC-MS), the UV kinetics resulted in less steep, parallel $\ln c$ versus $\mathrm{t}$ curves for all five chromophores, indicating similar rate constants (i.e. slopes) in this phase. This strongly suggested that the last-phase UV kinetics of all five compounds were governed by the slow degradation of the same formed secondary chromophore. The degradation rate of the secondary chromophore (same conditions as for HexA chromophores 1-5) is given for comparison in Fig. 2, being about 8 times slower than that of the "slowest" HexA chromophore to degrade, namely compound 5. With regard to that intermediate secondary chromophore, the degradation of the HexA chromophores 1-5 was about 25, 32, 202, 114 and 8 times faster, respectively; and while the half-lives for the HexA chromophores are between 5 and $120 \mathrm{~s}$, the one of the intermediate is more than 16 min (Table 1).

The temperature dependence of the kinetic rate constant for the chromophore degradation was determined by recording the degradation kinetics (UV) at different temperatures in $10{ }^{\circ} \mathrm{C}$ steps from 10 up to $70{ }^{\circ} \mathrm{C}$. We used only the initial reaction rates, up to conversions of about $20 \%$, to be sure that the kinetics were not significantly influenced by the formation of the intermediate. From these data, the Arrhenius activation energy $E_{A}$ was calculated, which is defined to be $(-1 / R)$ times the slope of a plot of $\ln (k)$ versus $(1 /$ $T$ ), and the activation parameters for the degradation were calculated. The respective kinetic data are summarized in Table 2.

The activation energies $\left(E_{A}, \Delta \mathrm{H}^{*}\right.$ and $\left.\Delta \mathrm{G}^{\ddagger}\right)$ are in the expected region, as well as the pre-exponential Arrhenius factor, which is related to the activation entropy (Atkins 1990). The Arrhenius and Eyring activation energies are connected through the equation $\mathrm{E}_{\mathrm{A}}=\Delta \mathrm{H}^{\star}+\mathrm{RT}$. The $\Delta \mathrm{H}^{\ddagger}$ values thus reflect the same order of compounds as the kinetic rate constants. Compound 5 with the highest activation energy of the five chromophores had the lowest kinetic rate constant. All five chromophore reactions had negative activation entropies, $\left(\Delta S^{*}\right)$, showing that the transition state of the rate-determining step is of higher order than the isolated reactants. Assuming that the attack of hydrogen peroxide (or its anion) at the chromophore is rate-determining, there is certainly a considerable entropy loss by bringing the two molecules together in one transition complex with concomitant loss of rotational and translational degrees of freedom.

Table 1 Degradation of the HexA-derived chromophores 1-5 and the intermediate DHBQ by alkaline $\mathrm{H}_{2} \mathrm{O}_{2}$ at $70{ }^{\circ} \mathrm{C}$

\begin{tabular}{llcll}
\hline Compound & $k\left(\mathrm{~s}^{-1}\right)$ & $\tau_{1 / 2}(\mathrm{~s})$ & Rate rel. to $\mathbf{5}$ & Rate rel. to DHBQ \\
\hline $\mathbf{1}$ & $1.75 \times 10^{-2}$ & 39.6 & 3.1 & 24.6 \\
$\mathbf{2}$ & $2.26 \times 10^{-2}$ & 30. & 4.0 & 31.7 \\
$\mathbf{3}$ & $1.44 \times 10^{-1}$ & 4.8 & 25.6 & 202.2 \\
$\mathbf{4}$ & $8.13 \times 10^{-2}$ & 8.5 & 14.5 & 114.2 \\
$\mathbf{5}$ & $5.62 \times 10^{-3}$ & 123.3 & 1 & 7.9 \\
DHBQ $(\mathbf{6})$ & $7.18 \times 10^{-4}$ & 973.5 & 0.126 & 1 \\
\hline
\end{tabular}

Rate constants $k\left(\mathrm{~s}^{-1}\right)$, half-times $\tau_{1 / 2}(\mathrm{~s})$ and relative reaction rates (rounded to the first decimal) 
Table 2 Activation parameters for the pseudo-first order degradation of chromophores 1-5 (and intermediate DHBQ) by alkaline hydrogen peroxide at $20^{\circ} \mathrm{C}(292.15 \mathrm{~K})$

\begin{tabular}{llllll}
\hline Compound & $E_{A}(\mathrm{kcal} / \mathrm{mol})$ & $A$ & $\Delta \mathrm{H}^{\ddagger}(\mathrm{kcal} / \mathrm{mol})$ & $\Delta \mathrm{S}^{\ddagger}[\mathrm{cal} /(\mathrm{mol} \mathrm{K})]$ & $\Delta \mathrm{G}^{\ddagger}(\mathrm{kcal} / \mathrm{mol})$ \\
\hline $\mathbf{1}$ & 13.8 & $2.32 \times 10^{11}$ & 13.2 & -8.5 & 15.7 \\
$\mathbf{2}$ & 14.1 & $2.24 \times 10^{11}$ & 13.5 & -8.6 & 16.0 \\
$\mathbf{3}$ & 11.9 & $8.05 \times 10^{10}$ & 11.3 & -10.6 & 14.4 \\
$\mathbf{4}$ & 11.7 & $6.64 \times 10^{10}$ & 11.1 & -11.0 & 14.3 \\
$\mathbf{5}$ & 15.0 & $2.52 \times 10^{11}$ & 14.4 & -8.3 & 16.8 \\
DHBQ (6) & 16.8 & $8.91 \times 10^{11}$ & 16.2 & -5.8 & 17.9 \\
\hline
\end{tabular}

$E_{A}$ Arrhenius activation energy, $A$ Arrhenius pre-exponential factor, $\Delta \mathrm{H}^{*}$ activation enthalpy, $\Delta \mathrm{S}^{\ddagger}$ activation entropy at $292.15 \mathrm{~K}$, $\Delta \mathrm{G}^{*}$ free activation energy (Gibbs activation energy) at $292.15 \mathrm{~K}$

Interestingly, the activation entropy was somewhatby about $2 \mathrm{cal} /(\mathrm{mol} \mathrm{K})$-more negative for $\mathbf{3}$ and $\mathbf{4}$ than for the other chromophores, indicating a higher order in the transition state. It is reasonable to assume that the carboxyl groups are involved in this organizational effect, either by ionic attraction or by accepting hydrogen bonds from attacking hydrogen peroxide (anions). The more negative activation entropy somewhat levels out the considerable activation energy differences $\left(\Delta H^{\star}\right)$ between 3 and 4 on one side and 1, 2 and $\mathbf{5}$ on the other side, according to the equation $\Delta \mathrm{G}^{\ddagger}=\Delta \mathrm{H}^{\star}-\mathrm{T} \times \Delta \mathrm{S}^{\ddagger}$, so that the values of free activation energies $\left(\Delta \mathrm{G}^{\ddagger}\right)$ of all five compounds are closer than those of the activation energies. The effects of the small negative activation entropy and the high activation energy for the reaction of DHBQ add to each other and account for the rather slow DHBQ consumption compared to chromophores 1-5.

\section{Degradation intermediates and products}

From the UV-detection curve (see Fig. 1) it was obvious that the degradation produced a secondary chromophore, with its UV absorption being in a similar range as that of the starting chromophore and thus evidently interfering with the measurement. While the starting chromophore was monotonously consumed according to first order-kinetics (see Fig. 2), the secondary chromophore, which was much slower degraded than the initial one, accumulated increasingly and governed the kinetics in the later and final phase of the reaction when the starting chromophore was already gone ( $c f$. UV vs. GC curve in Fig. 1).
In order to isolate the apparently quite stable intermediate-stable at least in comparison to the starting chromophores themselves-we stopped the degradation reactions at a conversion of $90 \%$ of the starting chromophores (measured by GC) by fast neutralization, freezing in liquid nitrogen and subsequent freezedrying. The remainders were separated by flash chromatography as described in the previous part, and the individual components were analyzed. With about $90 \%$ of the respective HexA chromophores being consumed, the highest concentration of intermediate relative to non-consumed starting material was supposed to be present, and it was likely that the amount of intermediate present was sufficient for identification and proper analytical characterization.

Indeed identification was quite easy by comparison with previous results, and two compounds, apart from non-converted starting material, were unambiguously identified by a combination of analytical techniques (NMR, GC-MS, FTIR, all also by spiking with authentic samples): 2,5-dihydroxy-[1,4]-benzoquinone (DHBQ, 6) was dominant in the mixtures from all five chromophores. In fact, it was the only intermediate compound identifiable when starting from either 1, 2 or 5. DHBQ is the most prominent one of the three key chromophores identified in diverse cellulosic materials (Rosenau et al. 2004, 2007; Korntner et al. 2015). In the case of compounds $\mathbf{3}$ and $\mathbf{4}$ as the starting materials, 2,5dihydroxyacetophenone (HAP, 7) was found in addition to DHBQ, but in significantly smaller amounts, approx. $10 \%$ of the DHBQ amount (see Scheme 2). HAP is another one of the three key chromophores in cellulosics (Rosenau et al. 2004, 2007; Korntner et al. 
Scheme 2 Degradation of the HexA-derived chromophores 1-5 under P-stage conditions, leading to the two intermediates DHBQ (6) and HAP (7), which are slower degraded than the starting compounds, and finally to colorless degradation products<smiles>O=C1c2ccoc2C(=O)c2ccoc21</smiles><smiles>O=C(O)c1cc2c(o1)C(=O)c1oc(C(=O)O)cc1C2=O</smiles><smiles>O=C1c2occc2C(=O)c2c1oc1c(C(=O)O)ccc(O)c21</smiles>

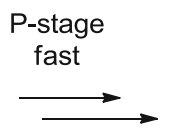<smiles>O=C1C=C([O-])C(=O)C([O-])=C1</smiles><smiles>CC(=O)O</smiles><smiles>O=CO</smiles>

3<smiles>O=C(O)c1ccc2oc3c(oc4c(C(=O)O)ccc(O)c43)c(=O)c2c1O</smiles><smiles>CC(=O)c1cc(O)ccc1[O-]</smiles>

$\mathrm{CO}_{2}$<smiles>O=c1c2ccoc2c(=O)c2c1oc1c(=O)c3c(oc4c(=O)c5occc5c(=O)c43)c(=O)c12</smiles>

2015). An additional confirmation for the intermediate occurrence came from the kinetic experiments (see Fig. 2): the reaction rate constants in the final phase of the chromophore degradation, i.e. when only DHBQ was left, were the same (with an error of $4 \%$ ) as that determined previously for the alkaline degradation of pure 2,5-dihydroxy-[1,4]-benzoquinone under otherwise identical conditions. ${ }^{2}$

No chromophoric intermediates other than 6 and 7 were detected. When running the reaction in $\mathrm{D}_{2} \mathrm{O} /$ $\mathrm{NaOD}$, both acetic acid as well as formic acid—both

\footnotetext{
$\overline{2}$ In previous work (see Hosoya and Rosenau 2013 ), DHBQdegradation in alkaline $\mathrm{H}_{2} \mathrm{O}_{2}$ had been carried out at $\mathrm{pH} 10$. We have repeated this degradation at $\mathrm{pH} 12$, and also the degradation of compounds $\mathbf{1}, \mathbf{2}$ and $\mathbf{5}$ at $\mathrm{pH} 10$. At both $\mathrm{pH}$ values, the rate constant for the degradation of DHBQ and the rate constant for the second degradation phase of the HexA-chromophore were pairwise equal.
}

evidently non-chromophoric-were detected by NMR in the reaction mixtures of all five chromophores at above $60 \%$, and were additionally confirmed by spiking the mixture with the authentic compounds. The same two organic acids, namely acetic acid and formic acid, remained as the only detectable final organic compounds-besides inorganic carbonatewhen the overall reaction was driven to completion and also the intermediates were completely consumed (Scheme 2). This agrees very well with the previously established mechanism for $\mathrm{H}_{2} \mathrm{O}_{2}$ degradation of DHBQ under alkaline conditions that had shown these two acids-besides carbonate- to be the final degradation products of DHBQ (Hosoya and Rosenau 2013a).

The generation of DHBQ (and HAP) from the HexA chromophores might easily be disregarded as less relevant, but it is actually of high importance: the 


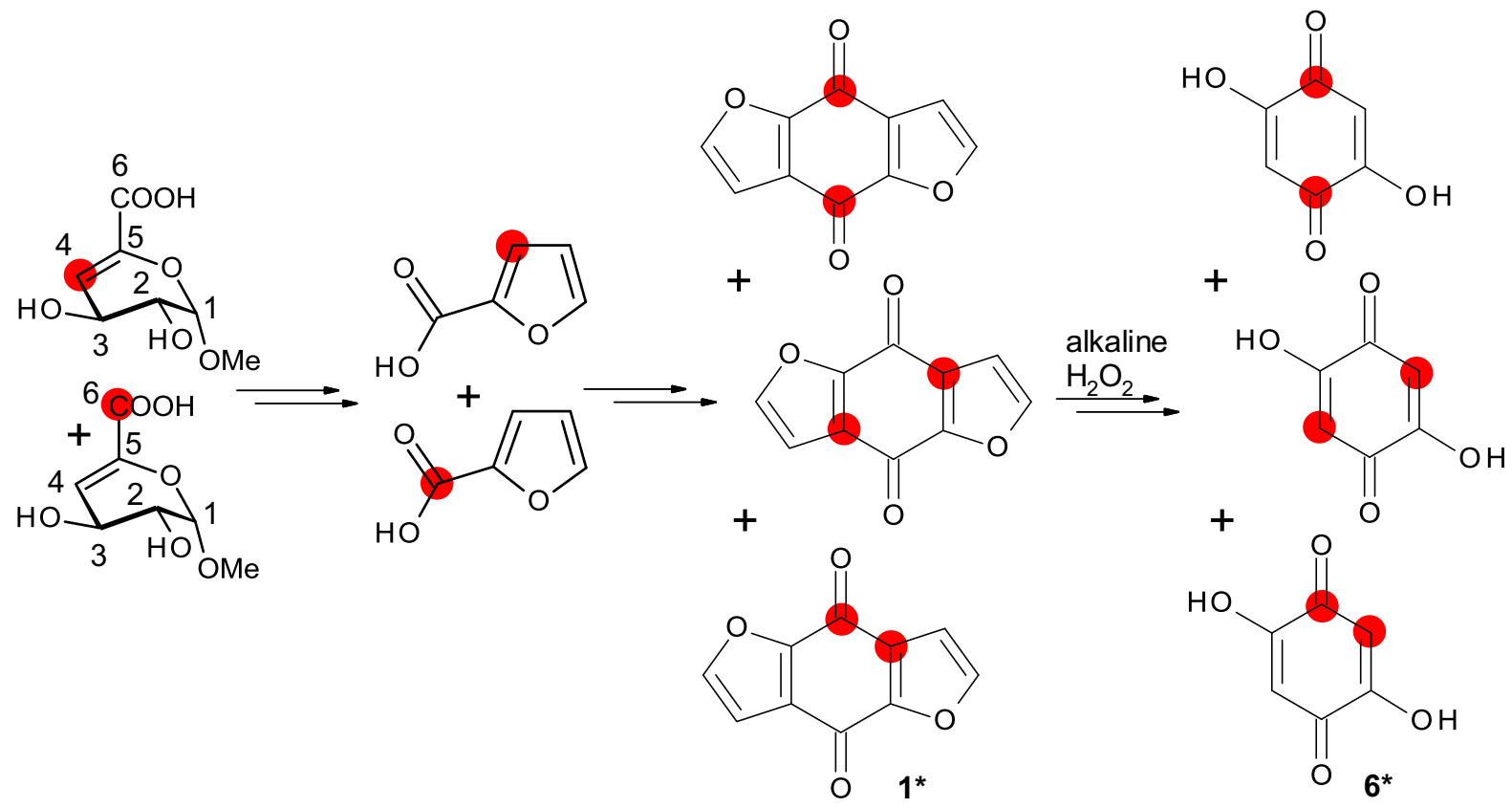

Scheme 3 A 1:1 mixture of differently ${ }^{13} \mathrm{C}$-labeled HexA model compounds provided $\mathbf{1}^{*}$ as a mixture of 3 isotopomers. The same pattern was found in the product, namely labeled DHBQ $\left(6^{*}\right)$

degradation of HexA moieties produces structurally interrelated compounds (1-5) which under P-stage conditions are all converted into the same common intermediates. These intermediates are more slowly degraded than any of the starting chromophores. Under circumstances of a $\mathrm{P}$ bleaching stage, the bleaching chemistry of the HexA chromophores is thus simply translated into that of DHBQ!.

DHBQ (6) is a very potent, strongly UV/Visabsorbing compound (Hosoya et al. 2013a), which represents the above-discussed secondary chromophore that has already been detected in the kinetic studies. The derived dianion, as the DHBQ species being present at $\mathrm{pH} 12$, experiences exceptional resonance stabilization, and the resulting delocalization of the double bonds explains its low reactivity towards bleaching agents that act on double bonds, such as hydrogen peroxide and ozone (Hosoya et al. 2013a). In aqueous solution it is of intensely dark blue color so that at concentration above $1 \mathrm{mM}$ it appears almost black. From the comparison of the kinetic rate constants ( $k$ values) above, degradation of DHBQ proceeded about 8-200 times slower than that of the HexA-derived chromophores 1-5 (cf. Table 1), which nicely illustrates the effect of the resonance stabilization.
In the next step, the question that needed to be answered was how DHBQ is formed from each of the HexA-derived compounds. Of course, 1,4-benzoquinone moieties contained in each of the compounds 1-5 suggested themselves as precursors to the formation of $\mathbf{6}$, but some doubts remained, e.g. with regard to the puzzling $\mathrm{C}-\mathrm{C}$ bond cleavage between this unit and the neighboring furan and the resulting $\mathrm{CH}$-structure in ring-positions 3 and 6 of DHBQ. A solid proof of the structures from which DHBQ originated was provided once more by the help of ${ }^{13} \mathrm{C}$-isotopic labeling, using the ${ }^{13} \mathrm{C}$-isotopomers described in the two preceding parts of this series. Schemes 3, 4, 5, 6 and 7 summarize the formation of ${ }^{13} \mathrm{C}$-labeled chromophores $(1 *-5 *)$ and their degradation under $\mathrm{P}$-stage conditions into ${ }^{13} \mathrm{C}$-labeled DHBQ. Labeling with ${ }^{13} \mathrm{C}$ is indicated by red dots marking the labeled position in the formulae, and by asterisks after the compound number. Formation and analysis of the isotopomers has been described in the two preceding parts of this series, which focused on the use of ${ }^{13} \mathrm{C}$-isotopic labeling in addressing the formation of the mixed benzoidfuranoid ladder-type oligomers from their primary (HexA) and secondary (furanoid) precursors. These formation reactions shall not be repeated here. The positions of the isotopic labels in DHBQ were highly 


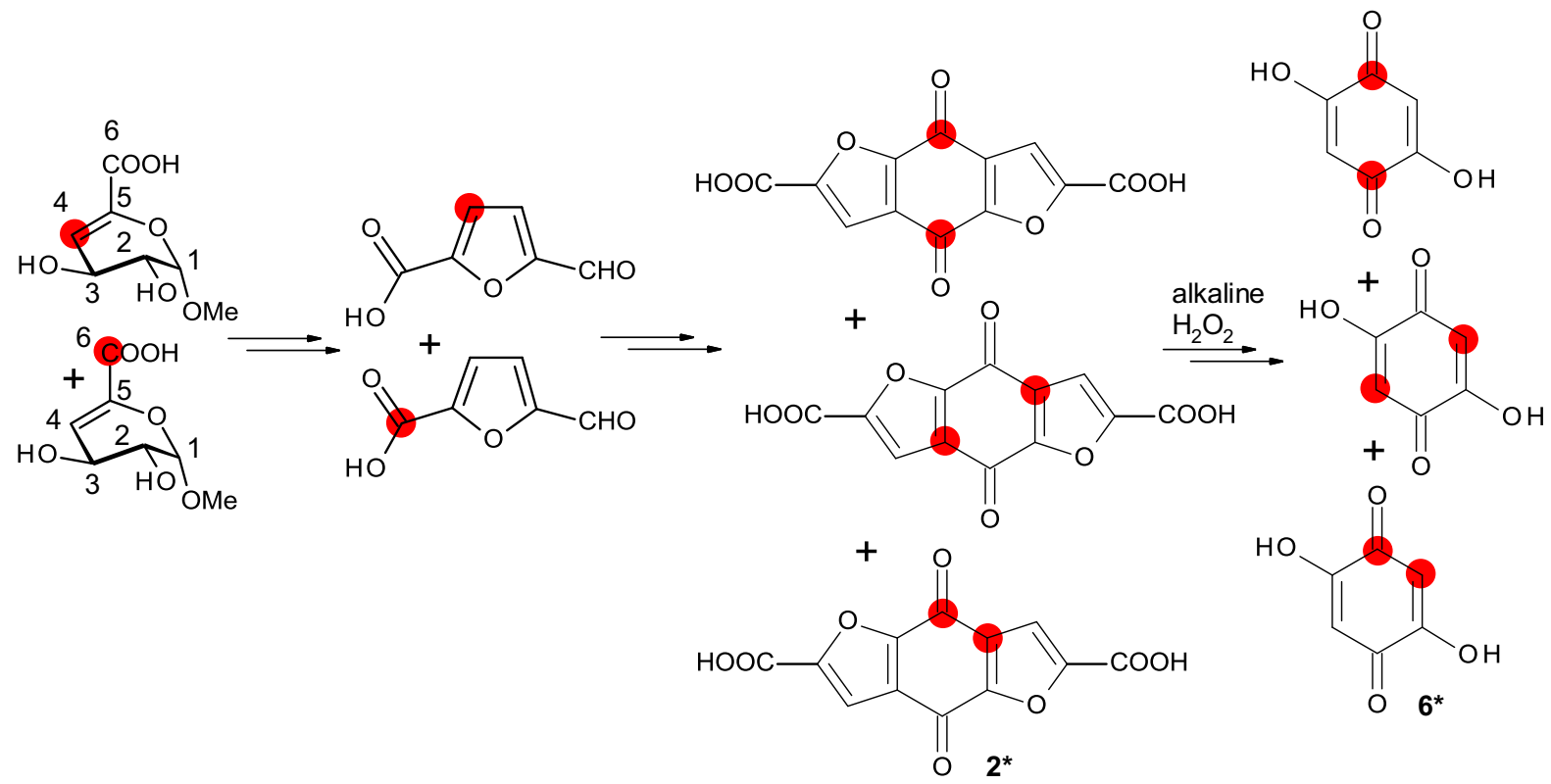

Scheme 4 A 1:1 mixture of differently ${ }^{13} \mathrm{C}$-labeled HexA model compounds provided $2 *$ as a mixture of 3 isotopomers. The same pattern was found in the product, namely labeled DHBQ $\left(\mathbf{6}^{*}\right)$. Note that the exocyclic carboxyl groups in $\mathbf{2}$ originate from $\mathrm{C}-1$ (not C-6) in the HexA model compound, which translates into the aldehyde groups in the resulting 5-formyl-2-furancarboxylic acids that undergo condensation and oxidation (see previous part of this series ${ }^{1}$ )

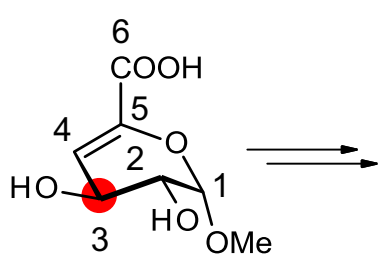<smiles>O=C(O)c1cccc2c1OC1C(=O)c3occc3C(=O)C21</smiles><smiles>C=CC(OC)[C@H]1O[C@@H](C(=O)O)[C@@H](C)[C@H]1O</smiles>

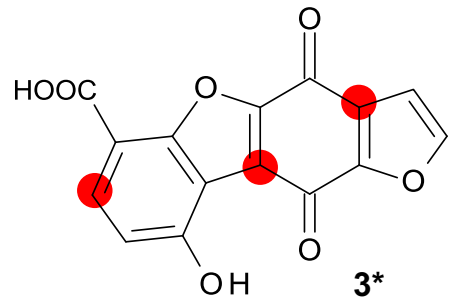

Scheme 5 A ${ }^{13}$ C-labeled HexA model compound provided $3 *$ with three labeled positions (top line), a different label position resulting in a different isotopic pattern in $3^{*}$ (bottom line). The

indicative of their "placement" in the original molecule. The isotopic pattern can be readily analyzed by ${ }^{13} \mathrm{C}$ NMR. Besides the evident signal enforcement at the labeled positions as the most prominent feature, also the occurrence of homonuclear $J_{\mathrm{C}-\mathrm{C}}$ couplings are<smiles>CC(=O)c1c(O)cccc1O</smiles><smiles>CC(=O)c1cc(O)[o+]cc1O</smiles>

isotopic pattern in the products $6 *$ and $7 *$ proved their origin in the $[1,4]$-benzoquinone moiety and in the $p$-hydroxybenzoate motif, respectively, of $3^{*}$

additional characteristics of high analytical value (see previous parts). In all five cases, the congruency between the $[1,4]$-benzoquinone moieties in the chromophores 1-5 and the produced DHBQ was confirmed. Degradation of the chromophores and 


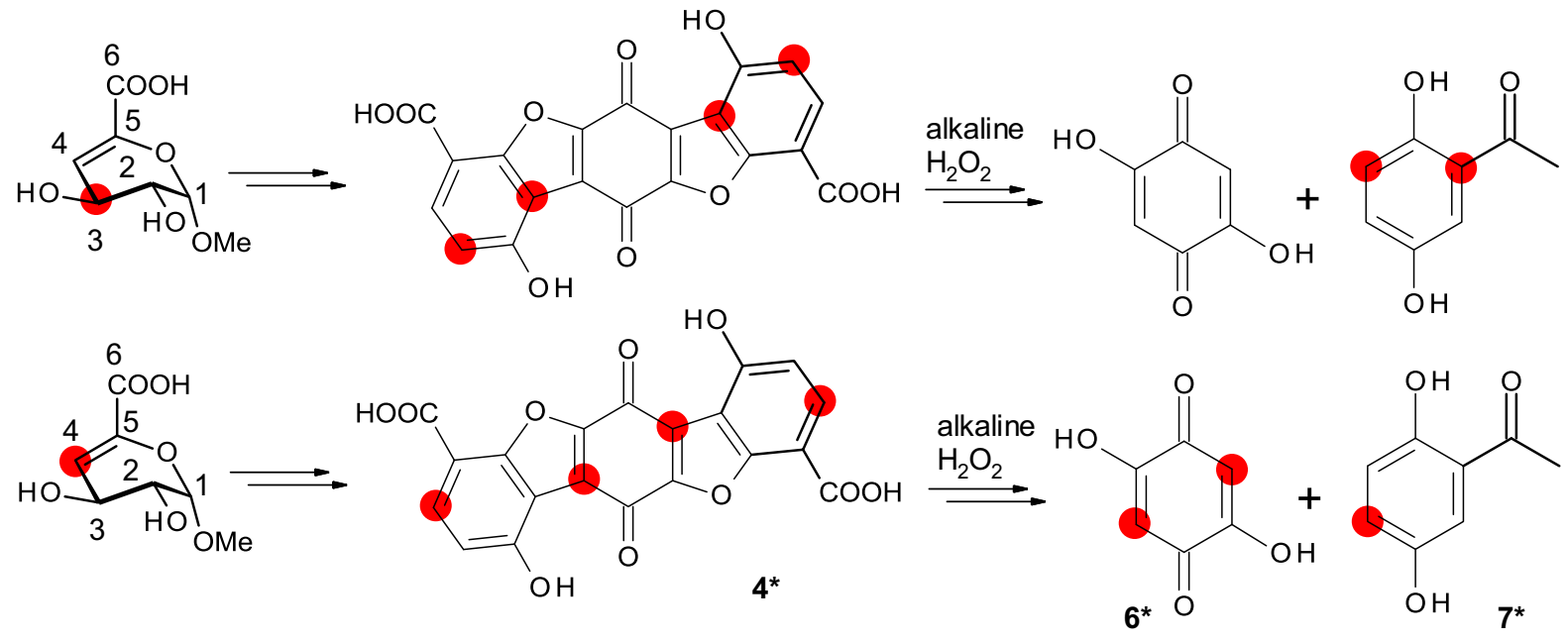

Scheme 6 A ${ }^{13}$ C-labeled HexA model compound provided 4* with four labeled positions (top line), a different label position resulting in a different isotopic pattern in $4 *$ (bottom line). The

isolation of the DHBQ isotopomers was performed as described above for the non-labeled compounds. The time for the stopping of the reaction should coincide with the maximum concentration of $\mathbf{6}$, especially in the case of the valuable isotopically labeled compounds. These times were either taken from the above-described kinetic studies or mathematically derived. $^{3}$

\section{Conclusion}

The five HexA-derived chromophores 1-5 are degraded by alkaline peroxide in pseudo-first order reactions at reaction rates between $1.44 \times 10^{-1}$ and $5.62 \times 10^{-3} \mathrm{~s}^{-1}$. Compound $\mathbf{3}$, the fastest compound to degrade, reacted about 25 times faster than compound $\mathbf{5}$, which was observed to be the slowest in this degradation system. All compounds form the same intermediate, namely DHBQ (6), derived from the substituted 1,4-benzoquinone moieties in all of the five compounds. By means of ${ }^{13} \mathrm{C}$-isotopically labeled HexA chromophores, these structural motifs in the

\footnotetext{
${ }^{3}$ If both production and consumption of $\mathbf{6}$ proceed according to first-order kinetics, a system $\mathrm{A} \rightarrow \mathrm{B} \rightarrow \mathrm{C}$ with $\mathrm{k}_{\mathrm{a}}$ for $\mathrm{A} \rightarrow \mathrm{B}$ and $\mathrm{k}_{\mathrm{b}}$ for $\mathrm{B} \rightarrow \mathrm{C}$ is obtained. The concentration of $\mathrm{B}$ is defined by: $d[B] / d t=k_{a} *[A]-k_{b} *[B]$. The concentration of $B$ (i.e. intermediate 6) is given by: $[\mathrm{B}]=\mathrm{K}_{\mathrm{a}}{ }^{*}\left[\mathrm{~A}_{0}\right] /\left(\mathrm{k}_{\mathrm{b}}-\mathrm{k}_{\mathrm{a}}\right)^{*}$ $\left(\mathrm{e}^{\left(-\mathrm{k}_{\mathrm{a}} * \mathrm{t}\right)}-\mathrm{e}^{\left(-\mathrm{k}_{\mathrm{b}} * \mathrm{t}\right)}\right)$. The maximum concentration of $\mathrm{B}$ (first derivative $=0)$ is reached at: $\mathrm{t}_{\max }=\ln \left(\mathrm{k}_{\mathrm{a}} / \mathrm{k}_{\mathrm{b}}\right) /\left(\mathrm{k}_{\mathrm{a}}-\mathrm{k}_{\mathrm{b}}\right)$.
}

isotopic pattern in the products $6 *$ and $7 *$ proved their origin in the $[1,4]$-benzoquinone moiety and in the $p$-hydroxybenzoate motifs, respectively, of $4 *$

HexA chromophores were confirmed as the origin of DHBQ generation. Besides this main intermediate small amounts of HAP (7)—about $10 \%$ of formed DHBQ-were formed as initial product from compounds 3 and 4 only, resulting from the anellated $p$ hydroxybenzoate moieties in the parent chromophores.

Both DHBQ (6) and HAP (7) represent two of the three key chromophores that are nearly ubiquitous in cellulosic matrices (Korntner et al. 2015). These two compounds are formed as intermediates by treatment of the HexA chromophores under P-stage conditions, and are, in turn, further converted at a rate about 8-200 times slower than the initial chromophores. This account thus provided the logical chemical link between HexA chemistry and the residual key chromophores in yellowing and brightness conversion of pulps. Properties and chemical behavior of those three key chromophores have recently been reviewed and shall therefore not be repeated here (Hosoya et al. 2013a; b, 2015).

There are two interesting conclusions from this part of the series: first, under P-stage conditions, the bleaching chemistry of the five HexA-chromophores turns into that of DHBQ and HAP, as already stated above. Second, DHBQ and HAP as key chromophores in pulp are indicators of some "HexA history" of the respective pulp, while the third key chromophore, 5,8dihydroxy-[1,4]-naphthoquinone (DHNQ), is evidently not linked to HexA chemistry. In other words, 


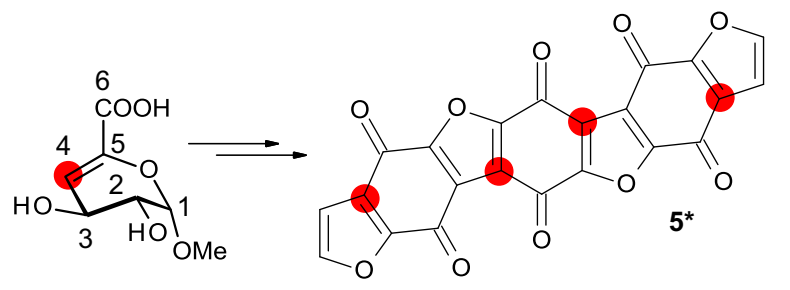

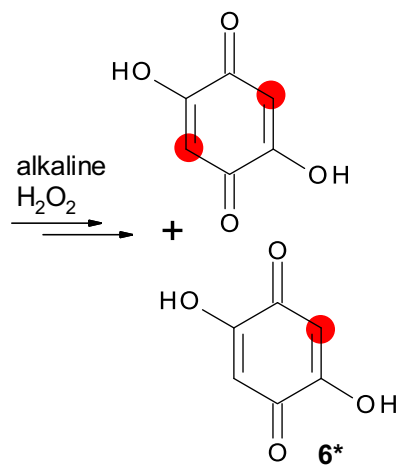

Scheme 7 A ${ }^{13}$ C-labeled HexA model compound provided $5^{*}$ with four labeled positions, which provided labeled DHBQ $\left(\mathbf{6}^{*}\right)$ with two different isotopic patterns. Note that the experimental ratio between dilabeled $6^{*}$ and monolabeled $6^{*}$ was about 2.5:1 and thus different from the theoretical ratio of $1: 2$, indicating that the lateral $[1,4]$-benzoquinone motifs (giving monolabeled
DHBQ) in $\mathbf{5}^{*}$ are degraded more quickly (or according to a different mechanism) than the central unit (giving dilabeled DHBQ). The labeling pattern in the DHBQ isotopomeric mixture can be readily analyzed, as dilabeled $6^{*}$ has a homonuclear ${ }^{3} J_{\mathrm{C}-\mathrm{C}}$ coupling of $8 \mathrm{~Hz}$ (signal as a doublet), while monolabeled $6 *$ offers no such feature (singlet resonance)

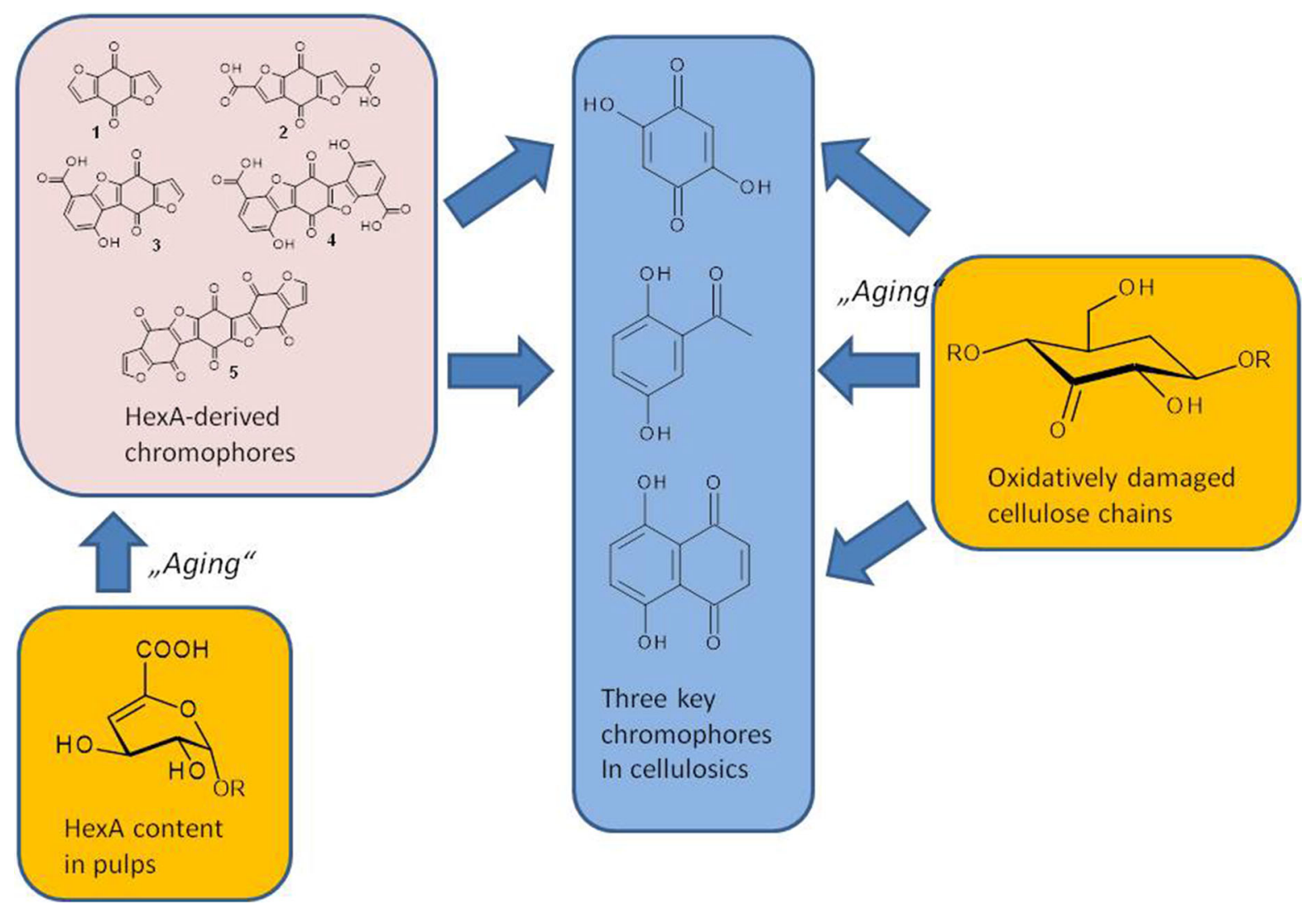

Scheme 8 Schematic illustration of the interrelation between HexA-derived chromophores, oxidatively dameged cellulose and the three key chromophores in cellulosic matrices. "Aging" denotes, chemical, thermal or irradiation stress. $\mathrm{R}$ denotes the truncated chains in xylan and cellulose, respectively 
while all three cellulosic key chromophores have one possible origin in the oxidative damage of cellulose (Lachenal and Chirat 1998; Potthast et al. 2005), only two of them-DHBQ and HAP, but not DHNQ - are also connected with HexA. For a pulp, in which DHNQ dominates among the residual chromophores, a contribution of HexA to yellowing and brightness reversion can thus be excluded; and a pulp in which DHBQ and HAP dominate over DHNQ is likely to have HexA as a major chromophore source, in addition to some possible oxidative damage of cellulose. The relations between the three key chromophores and possible causes of yellowing and brightness reversion are schematically shown in Scheme 8.

With the studies regarding the chemistry of HexAderived chromophores, as put forward in this part and the two preceding accounts of the chromophore paper series, we have hoped to contribute to a better understanding of bleaching chemistry, which might result in better bleaching performances, milder conditions and chemical savings, and thus eventually in environmentally more benign pulp processing and "greener" products.

Acknowledgments Open access funding provided by University of Natural Resources and Life Sciences Vienna (BOKU). The authors would like to thank the Austrian Forschungsförderungsgesellschaft (FFG) for financial support through project 847169 ("Chromophores in cellulosic pulps II") and through the project FLIPPR ${ }^{2}$ (Future Lignin and Pulp Processing Research), along with its partner companies. We are indebted to the partner companies in this project for providing cellulosic pulps, valuable input to every-day pulping and bleaching practice as well as interesting discussions.

Open Access This article is distributed under the terms of the Creative Commons Attribution 4.0 International License (http:// creativecommons.org/licenses/by/4.0/), which permits unrestricted use, distribution, and reproduction in any medium, provided you give appropriate credit to the original author(s) and the source, provide a link to the Creative Commons license, and indicate if changes were made.

\section{References}

Atkins PW (ed) (1990) Physikalische chemie. VCH Verlagsgesellschaft mbH, Weinheim

Azevedo MAB, Pasa VMD, Hamalainen H, Mounteer AH, de Oliveira RC, Colodette JL (2011) ECF bleaching with molybdenum activated acid peroxide and its impact on eucalyptus pulp properties and effluent quality. Nat Resour 2:61-70
Buchert J, Teleman A, Harjunpaa V, Tenkanen M, Viikari L, Vuorinen T (1995) Effect of cooking and bleaching on the structure of xylan in conventional pine kraft pulp. Tappi J 78(11):125-130

Chakar FS, Allison L, Ragauskas AJ, McDonough TJ (2000) Influence of hexenuronic acids on US bleaching operations. Tappi J 83(11):62

Chenna NK, Jääskelainen AS, Vuorinen T (2013) Rapid and selective catalytic oxidation of hexenuronic acid and lignin in cellulosic fibers. Ind Eng Chem Res 52:17744-17749

Clayton DW (1963) The alkaline degradation of some hardwood 4- $O$-methyl-D-glucuronoxylans. Sven Papperstidn 66:115-124

Hosoya T, Rosenau T (2013a) Degradation of 2,5-Dihydroxy1,4-benzoquinone by hydrogen peroxide under moderately alkaline conditions resembling pulp bleaching: a combined kinetic and computational study. J Org Chem 78(22):11194-11203

Hosoya T, Rosenau T (2013b) Degradation of 2,5-Dihydroxy1,4-benzoquinone by hydrogen peroxide: a combined kinetic and theoretical study. J Org Chem 78(7):3176-3182

Hosoya T, French AD, Rosenau T (2013a) Chemistry of 2,5dihydroxy-[1,4]-benzoquinone, a key chromophore in aged cellulosics. Mini-Rev Org Chem 10(3):309-315

Hosoya T, French AD, Rosenau T (2013b) Chemistry of 5,8dihydroxy-[1,4]-naphthoquinone, a key chromophore in aged cellulosics. Mini-Rev Org Chem 10(3):302-308

Hosoya T, French AD, Rosenau T (2015) Chemistry of 2,5dihydroxyacetophenone, a key chromophore in cellulosic substrates. Mini-Rev Org Chem 12(1):88-95

Korntner P, Hosoya T, Dietz T, Eibinger K, Reiter H, Spitzbart M, Roeder T, Borgards A, Kreiner W, Mahler AK, Winter H, Groiss Y, French AD, Henniges U, Potthast A, Rosenau $\mathrm{T}$ (2015) Chromophores in lignin-free cellulosic materials belong to three compound classes. Chromophores in cellulosics, XII. Cellulose 22(2):1053-1062

Lachenal D, Chirat C (1998) High temperature chlorine dioxide delignification: a breakthrough in ECF bleaching of hardwood kraft pulps. TAPPI Press, Atlanda, pp 601-604

Petit-Breuilh X, Zaror C, Melo R (2004) Hexenuronic acid removal from unbleached kraft eucalyptus pulp by peroxymonosulfuric acid. J Chil Chem Soc 49:355-360

Potthast A, Rosenau T, Kosma P, Saariaho A-M, Vuorinen T (2005) On the nature of carbonyl groups in cellulosic pulps. Cellulose 12(1):43-50

Rosenau T, Potthast A, Milacher W, Hofinger A, Kosma P (2004) Isolation and identification of residual chromophores in cellulosic materials. Polymer 45(19):6437-6443

Rosenau T, Potthast A, Kosma P, Suess HU, Nimmerfroh N (2007) Isolation and identification of residual chromophores from aged bleached pulp samples. Holzforschung 61(6):656-661

Rosenau T, Potthast A, Krainz K, Yoneda Y, Dietz T, Shields ZPI, French AD (2011) Chromophores in cellulosics, VI. First isolation and identification of residual chromophores from aged cotton linters. Cellulose 18(6):1623-1633

Rosenau T, Potthast A, Zwirchmayr NS, Hettegger H, Plasser F, Hosoya T, Bacher M, Krainz K, Dietz T (2017) Chromophores from hexeneuronic acids-identification of HexA-derived chromophores. Cellulose (in press) 
Suess HU (2010) Pulp bleaching today. Walter de Gruyter $\mathrm{GmbH} \& \mathrm{Co} . \mathrm{KG}$, Berlin

Teleman A, Hausalo T, Tenkanen M, Vuorinen T (1996) Identification of the acidic degradation products of hexenuronic acid and characterization of hexenuronic acid-substituted xylooligosaccharides by NMR spectroscopy. Carbohydr Res 280(2):197-208 